\title{
Influence of alkene substituent in dictating the reaction course to form carbocycles or oxacycles during ring closing metathesis of acyclic trienes
}

\author{
RITABRATA DATTA and SUBRATA GHOSH* (1) \\ Department of Organic Chemistry, Indian Association for the Cultivation of Science, Jadavpur, Kolkata, \\ West Bengal 700 032, India \\ E-mail: ocsg@iacs.res.in
}

MS received 4 September 2017; accepted 2 October 2017; published online 6 November 2017

\begin{abstract}
Ring closing metathesis of acyclic trienes that can provide oxacycles or carbocycles has been investigated. It was found that a substituent on one of the alkene units determines the reaction course to provide either oxacycles or carbocyles exclusively irrespective of the ring size of the resulting compounds.
\end{abstract}

Keywords. Substituent effects; cyclization; carbocycles; oxygen heterocycles.

\section{Introduction}

Olefin metathesis emerged as a powerful tool for carbon-carbon bond formation. Of the various metathetic processes ring closing metathesis $(\mathrm{RCM})^{1}$ is the most extensively used reaction for the construction of carbo- as well as heterocycles. With the advent of large number of catalysts it has been possible to construct rings of various sizes having a variety of functional groups. RCM reaction has been found to be influenced to a considerable extent by a number of structural parameters. ${ }^{2}$ The size and the nature of the rings (carbo- or heterocycles) to be formed and the substituent on the alkene unit generally determines the outcome of the ring closure event.

As part of our continued interest ${ }^{3}$ in the application of RCM in the synthesis of natural products, we undertook a program for the synthesis of highly bioactive marine polyketide plakilactones ${ }^{4}$ such as plakilactone $\mathrm{C} \mathbf{1}$ using RCM as the key step. Structurally plakilactones possess a substituted butenolide moiety with an alkenyl chain at C-5. We anticipated that cross metathesis of an appropriate alkene unit with the butenolide 2 would afford 1 (Scheme 1). RCM of the acyclic triene 3 would, in principle, form the dihydrofuran derivative 4 which on

\footnotetext{
*For correspondence
}

oxidation will provide the butenolide 2 . Success of this strategy thus depends on RCM of the triene $\mathbf{3}$ to afford exclusively the dihydrofuran 4.

RCM of acyclic systems with multiple alkene units that may lead to carbo- and oxacycles is relatively less explored. It was reported ${ }^{5}$ that RCM of the tetraene 5 having bis-allyl ether moieties with Grubbs' 1st generation catalyst G-I at $60^{\circ} \mathrm{C}$ produced exclusively the oxacycle 6. Similarly, the hexaene 7 in presence of Grubbs' 2nd generation catalyst G-II at $70^{\circ} \mathrm{C}$ gave the oxacycle 8 exclusively (Scheme 2), although in these polyolefinic substrates there were possibilities for the formation of carbocycles.

In order to find out the feasibility of the formation of oxacycle 4 from RCM of the triene $\mathbf{3}$, we initially decided to investigate RCM of a series of trienes $9 \mathbf{a}-\mathbf{h}$, structural analogues of the triene 3 . RCM of the trienes $\mathbf{9 a - h}$ may proceed in three different modes to produce either the carbocycles $\mathbf{1 0}$ involving the double bonds ' $a$ ' and 'b' (path 1) or the oxacycles $\mathbf{1 1}$ involving the double bonds ' $b$ ' and 'c' (path 2) or the oxepenes 12 involving the alkene units 'a' and 'c' (path 3) (Scheme 3). We herein report the results of this investigation demonstrating that the absence or presence of an alkyl substituent on one of the alkene units determines the course of the RCM reaction producing exclusively either the carbocycles $\mathbf{1 0}$ or the oxacycles $\mathbf{1 1}$ or $\mathbf{1 2}$. To the best of our knowledge there is no report on RCM of acyclic systems such as the trienes 9. 


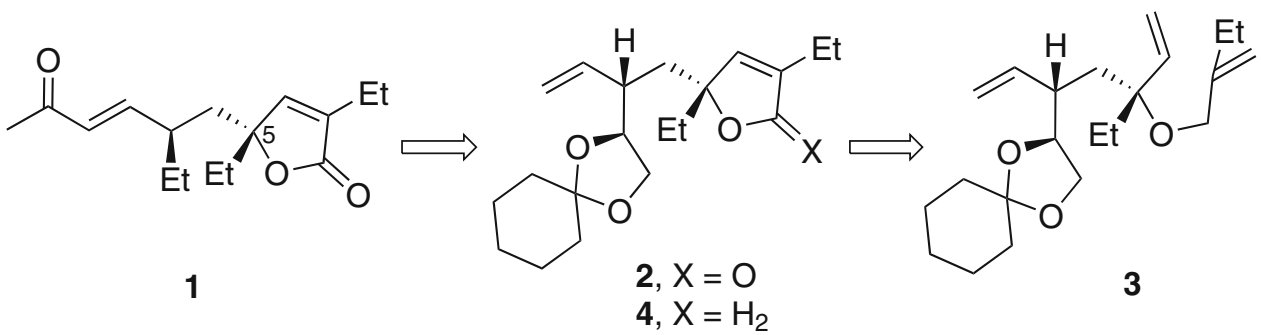

Scheme 1. Synthetic plan.<smiles>C=CCOC(CC=C)C(CC=C)OCC=C</smiles>

5<smiles>C=CCOC(CC=C)[C@H](C=C)O[C@H](C=C)C(CC=C)OCC=C</smiles>

7<smiles>CC(C)(Cl)CC1CC=CCO1</smiles><smiles>C1=CCC([C@@H]2C=C[C@H](C3CC=CCO3)O2)OC1</smiles>

8
$\mathrm{Cl}_{2}\left(\mathrm{PCy}_{3}\right)_{2} \mathrm{Ru}=\mathrm{CHPh}$

G I<smiles></smiles>

G ॥

Scheme 2. Reported RCM of polyenes.

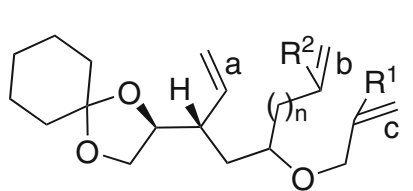

9a, $\mathrm{n}=0, \mathrm{R}^{1}=\mathrm{Me}, \mathrm{R}^{2}=\mathrm{H}$

b, $n=0, R^{1}=R^{2}=H$

c, $n=1, R^{1}=R^{2}=H$

d, $n=1, R^{1}=M e, R^{2}=H$

e, $n=2, R^{1}=R^{2}=H$

f, $\mathrm{n}=2, \mathrm{R}^{1}=\mathrm{Me}, \mathrm{R}^{2}=\mathrm{H}$

g, $n=0, R^{1}=H, R^{2}=M e$

h, $n=1, R^{1}=H, R^{2}=M e$

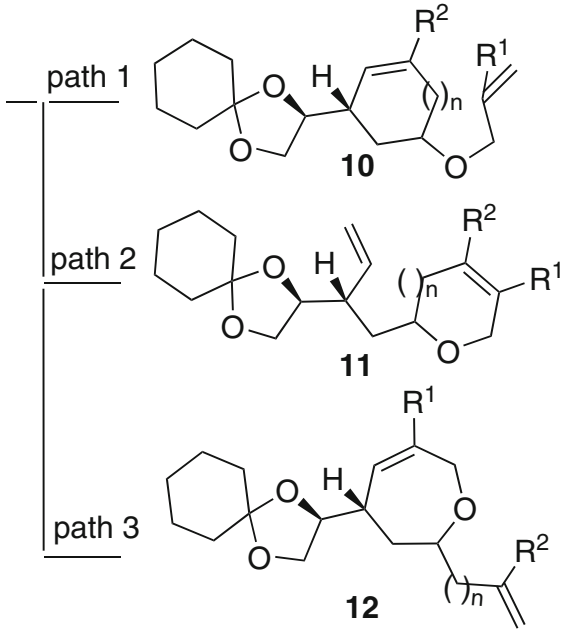

Scheme 3. Different possible routes in RCM of trienes.

\section{Experimental}

All air and moisture sensitive reactions were performed in oven dried glassware under argon atmosphere with freshly distilled anhydrous solvents. Reactions were monitored by thin layer chromatography (Silica gel $60 \mathrm{~F}_{254}$ ) with ethanolic anisaldehyde as developing agent. Organic extracts were dried over anhydrous sodium sulfate. Column chromatography was performed with 60-120 mesh silica gel unless otherwise stated. PE refers to the fraction of petroleum ether having B.p. $60-80{ }^{\circ} \mathrm{C}$. EA refers to ethyl acetate. Optical rotations were measured using sodium (589, D line) lamp and reported as follows: $[\alpha]_{\mathrm{D}}^{25}(\mathrm{c}=\mathrm{g} / 100 \mathrm{~mL}$, solvent). IR spectra for liquids were recorded as thin films. Unless otherwise stated NMR spectra were recorded in $\mathrm{CDCl}_{3}$ at $300 \mathrm{MHz}$ for ${ }^{1} \mathrm{H}$ and $75 \mathrm{MHz}$ for ${ }^{13} \mathrm{C}$ using residual chloroform as an internal standard and the chemical shifts are reported in $\delta \mathrm{ppm}$ scale. High resolution mass spectra (HRMS) were recorded on a QTOF I (quardrupole-hexapole-TOF) mass spectrometer with an orthogonal Z-spray-electrospray interface. 


\section{1 (S)-2-((3S,5S)-5-((2-Methylallyl)oxy)hepta-1,6-} dien-3-yl)-1,4-dioxaspiro[4.5]decane (9a)

A solution of the carbinol14 $(110 \mathrm{mg}, 0.44 \mathrm{mmol})$ in anhydrous THF ( $2 \mathrm{~mL}$ ) was added to a stirred suspension of sodium hydride (42 $\mathrm{mg}, 0.87 \mathrm{mmol}, 50 \%$ suspension in oil) in anhydrous $\mathrm{THF}(4 \mathrm{~mL})$ at $0{ }^{\circ} \mathrm{C}$. After $10 \mathrm{~min}, 3$-chloro2-methylpropene $(0.11 \mathrm{~mL}, 1.09 \mathrm{mmol})$ was added to it at $0^{\circ} \mathrm{C}$. The reaction mixture was stirred for $2 \mathrm{~h}$ at $\mathrm{rt}$ and was quenched with saturated aqueous ammonium chloride solution $(1 \mathrm{~mL})$. The reaction mixture was extracted with diethyl ether $(3 \times 20 \mathrm{~mL})$, washed with brine $(5 \mathrm{~mL})$, dried and concentrated. The residual mass was chromatographed $(4 \%$ EA/PE) to afford the triene $9 \mathbf{a}(77 \mathrm{mg}, 58 \%) ;[\alpha]_{\mathrm{D}}^{25}+11.0(c$ 6.3, $\left.\mathrm{CHCl}_{3}\right) ;{ }^{1} \mathrm{H}$ NMR $\delta$ 5.67-5.49 $(2 \mathrm{H}, \mathrm{m}), 5.22-5.01(4 \mathrm{H}$, $\mathrm{m}), 4.93-4.84(2 \mathrm{H}, \mathrm{m}), 3.95-3.87(3 \mathrm{H}, \mathrm{m}), 3.76-3.59(3 \mathrm{H}$, $\mathrm{m}), 2.27-2.17(1 \mathrm{H}, \mathrm{m}), 1.95-1.86(1 \mathrm{H}, \mathrm{m}), 1.71(3 \mathrm{H}, \mathrm{s})$, 1.69-1.63 (1H, m), 1.58-1.55 (8H, m), 1.40-1.34 (2H, m); ${ }^{13} \mathrm{C}$ NMR $\delta 142.7,138.7,138.2,118.0,117.3,111.7,109.7$, 79.0, 78.2, 71.9, 67.6, 44.9, 36.7, 36.6, 35.3, 25.3, 24.1, 24.0, 19.8; HRMS (ESI) $m / z$ calcd. for $\mathrm{C}_{19} \mathrm{H}_{30} \mathrm{O}_{3} \mathrm{Na}(\mathrm{M}+\mathrm{Na})^{+}$, 329.2093; Found, 329.2095.

\section{2 (S)-2-((1S,4S)-4-((2-Methylallyl)oxy)cyclopent- 2-en-1-yl)-1,4-dioxaspiro[4.5]decane (10a)}

The triene 9a (28 mg, $0.09 \mathrm{mmol})$ was taken in a degassed solution of dry toluene $(5 \mathrm{~mL})$ and then Grubbs 1 st generation catalyst G I (3.8 mg, $0.005 \mathrm{mmol}$ ) was added under argon atmosphere. The resulting mixture was stirred at $\mathrm{rt}$ for $12 \mathrm{~h}$ (TLC). The reaction mixture was concentrated under vacuum. Purification of the crude mixture by column chromatography (5\% EA/PE) afforded the carbocycle 10a (16 mg, 64\%) as colorless oil along with recovered starting material $(6 \mathrm{mg}$, $22 \%) ;[\alpha]_{\mathrm{D}}^{25}+119.9\left(c 2.9, \mathrm{CHCl}_{3}\right) ;{ }^{1} \mathrm{H} \mathrm{NMR} \delta 5.99(1 \mathrm{H}, \mathrm{dt}$, $J=2.1,5.7 \mathrm{~Hz}), 5.84(1 \mathrm{H}, \mathrm{ddd}, J=0.9,1.2,6.0 \mathrm{~Hz}), 4.97-$ 4.87 (2H, m), 4.63-4.57 (1H, m), 4.01-3.88 (4H, m), 3.60$3.53(1 \mathrm{H}, \mathrm{m}), 3.11-3.06(1 \mathrm{H}, \mathrm{m}), 2.02-1.95(2 \mathrm{H}, \mathrm{m}) 1.74$ $(3 \mathrm{H}, \mathrm{s}), 1.62-1.51(8 \mathrm{H}, \mathrm{m}), 1.43-1.32(2 \mathrm{H}, \mathrm{m}) ;{ }^{13} \mathrm{C}$ NMR $\delta$ $142.8,135.5,133.7,112.1,109.7,83.6,78.3,72.8,67.4,48.2$, 36.4, 35.1, 33.2, 25.4, 24.2, 24.0, 19.8; HRMS (ESI) $m / z$ calcd. for $\mathrm{C}_{17} \mathrm{H}_{26} \mathrm{O}_{3} \mathrm{Na}(\mathrm{M}+\mathrm{Na})^{+}$, 301.1780; Found, 301 . 1782.

\section{3 (6S)-6-((S)-1,4-Dioxaspiro[4.5]decan-2-yl)octa- 1,7-dien-4-ol (15)}

To a magnetically stirred solution of the aldehyde 13 (530 mg, $2.36 \mathrm{mmol})$ in dry THF $(15 \mathrm{~mL})$ cooled to $0^{\circ} \mathrm{C}$, allylmagnesium chloride (1.54 mL, $3.07 \mathrm{mmol}, 2 \mathrm{M}$ in THF) was added. The reaction mixture was stirred for $12 \mathrm{~h}$ and then quenched by saturated aqueous solution of $\mathrm{NH}_{4} \mathrm{Cl}$. The clear solution was filtered and concentrated. The residual mass was chromatographed (15\% EA-PE) as the eluent to afford the alcohol 15 (592 mg, 94\%) as a light yellow oil; ${ }^{1} \mathrm{H}$ NMR (of the mixture) $\delta 5.90-5.77(1 \mathrm{H}, \mathrm{m}), 5.66-5.46(1 \mathrm{H}, \mathrm{m})$, 5.14-5.04 (4H, m), 3.94-3.82 (2H, m), 3.72-3.59 (2H, m), $2.68(\mathrm{~s}) \& 2.55(\mathrm{~s})(1 \mathrm{H}), 2.40-2.31(1 \mathrm{H}, \mathrm{m}), 2.26-2.19(2 \mathrm{H}$, $\mathrm{m}), 1.84-1.73(1 \mathrm{H}, \mathrm{m}), 1.61-1.57(9 \mathrm{H}, \mathrm{m}), 1.40-1.21(2 \mathrm{H}$, $\mathrm{m}) ;{ }^{13} \mathrm{C}$ NMR (of the mixture) $\delta 138.3,138.1,135.1,135.0$, 117.8, 117.7, 117.3, 117.1, 110.1 (X 2), 78.4, 78.1, 69.3, 69.1, $68.3,68.2,46.5,45.8,42.8,41.5,39.9,39.8,36.6,36.5,35.3$ (X 2), 25.2 (X 2), 24.2, 24.1, 24.0 (X 2); HRMS (ESI) $\mathrm{m} / z$ calcd. for $\mathrm{C}_{16} \mathrm{H}_{26} \mathrm{O}_{3} \mathrm{Na}(\mathrm{M}+\mathrm{Na})^{+}$, 289.1780; Found, 289 . 1783.

\section{4 (2S)-2-((3S)-5-(Allyloxy)octa-1,7-dien-3-yl)-1,4- dioxaspiro[4.5]decane $(\mathbf{9 c})$}

Following the same synthetic procedure as described in the preparation of 9a, the carbinol $15(70 \mathrm{mg}, 0.26 \mathrm{mmol})$ was converted to the triene 9c $(66 \mathrm{mg}, 83 \%$, purified by column chromatography, 4\% EA/PE as eluent) by using sodium hydride (19 mg, $0.39 \mathrm{mmol}, 50 \%$ suspension in oil) and allyl bromide $(0.05 \mathrm{~mL}, 0.53 \mathrm{mmol}) ;{ }^{1} \mathrm{H} \mathrm{NMR}$ (of the mixture)

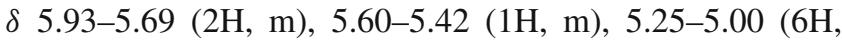
$\mathrm{m}), 4.07-3.80(4 \mathrm{H}, \mathrm{m}), 3.66-3.56(1 \mathrm{H}, \mathrm{m}), 3.47-3.35(1 \mathrm{H}$, m), 2.49-2.15 (3H, m), 1.98-1.81 (1H, m), 1.59-1.50 (9H, $\mathrm{m}), 1.38-1.22(2 \mathrm{H}, \mathrm{m}) ;{ }^{13} \mathrm{C} \mathrm{NMR}$ (of the mixture) $\delta 138.4$, 138.3, 135.5, 135.4, 134.8, 134.7, 117.6, 117.1, 117.0, 116.8, 116.4, 116.2, 109.7, 109.6, 78.3, 78.1, 76.9, 76.1, 69.9, 69.8, 67.6, 67.5, 45.5, 45.0, 38.9, 37.9, 36.6, 36.4, 35.9, 35.7, 35.3, 35.2, 25.3 (X 2), 24.1 (X 2), 24.0, 23.9; HRMS (ESI) $m / z$ calcd. for $\mathrm{C}_{19} \mathrm{H}_{30} \mathrm{O}_{3} \mathrm{Na}(\mathrm{M}+\mathrm{Na})^{+}, 329.2093$; Found, 329 . 2096.

\section{5 (2S)-2-((3S)-5-((2-Methylallyl)oxy)octa-1,7-dien- 3-yl)-1,4-dioxaspiro[4.5]decane (9d)}

Following the same allylation procedure as described in the preparation of 9a, the allyl alcohol15 $(150 \mathrm{mg}, 0.56 \mathrm{mmol})$ was converted to the triene 9d (116 mg, $65 \%$, purified by column chromatography, $4 \% \mathrm{EA} / \mathrm{PE}$ as eluent) by using sodium hydride (54 mg, $1.13 \mathrm{mmol}, 50 \%$ suspension in oil) and 3chloro-2-methylpropene $(0.14 \mathrm{~mL}, 1.41 \mathrm{mmol}) ;{ }^{1} \mathrm{H}$ NMR (of the mixture) $\delta 5.88-5.70(1 \mathrm{H}, \mathrm{m}), 5.61-5.42(1 \mathrm{H}, \mathrm{m}), 5.08$ $5.01(4 \mathrm{H}, \mathrm{m}), 4.93(1 \mathrm{H}, \mathrm{s}), 4.81(1 \mathrm{H}, \mathrm{s}), 3.96-3.83(4 \mathrm{H}, \mathrm{m})$, 3.64-3.59 (1H, m), 3.45-3.36 (1H, m), 2.46-2.19 (4H, m), 1.92-1.82 (2H, m), 1.71 (3H, s), 1.57-1.54 (6H, m), 1.371.33 (3H, m, ); ${ }^{13} \mathrm{C} \mathrm{NMR}$ (of the mixture) $\delta 142.8$ (X 2), 138.4, $138.3,134.9$ (X 2), 117.5, 117.1, 116.9, 116.8, 111.8, 111.6, 109.6 (X 2), 78.3, 78.2, 76.7, 76.2, 72.8, 72.7, 67.6 (X 2), $45.4,45.1,38.8,37.9,36.6,36.4,35.9,35.5,35.3,35.2,25.3$ (X 2), 24.1 (X 2), 23.9 (X 2), 19.8, 19.7; HRMS (ESI) $m / z$ calcd. for $\mathrm{C}_{20} \mathrm{H}_{32} \mathrm{O}_{3} \mathrm{Na}(\mathrm{M}+\mathrm{Na})^{+}, 343.2249$; Found 343 . 2247.

\section{6 (2S)-2-((3S)-5-(Allyloxy)nona-1,8-dien-3-yl)-1,4- dioxaspiro[4.5]decane $(\mathbf{9 e})$}

Following the same synthetic procedure as described in the preparation of 9a, the carbinol $16(105 \mathrm{mg}, 0.37 \mathrm{mmol})$ was 
converted to the triene $9 \mathrm{e}$ (94 $\mathrm{mg}, 80 \%$, purified by column chromatography, $4 \%$ EA/PE as eluent) by using sodium hydride (27 mg, $0.56 \mathrm{mmol}, 50 \%$ suspension in oil) and allyl bromide $(0.07 \mathrm{~mL}, 0.74 \mathrm{mmol}) ;{ }^{1} \mathrm{H}$ NMR (of the mixture) $\delta$ 5.96-5.73 (2H, m), 5.60-5.45 (1H, m), 5.27-4.91 (6H, m), 4.04-3.84 (4H, m), 3.68-3.35 (2H, m), 2.47-2.38 (1H, m), 2.22-2.04 (3H, m), 1.97-1.80 (1H, m), 1.59-1.55 (10H, m), 1.38-1.34 (2H, m); ${ }^{13} \mathrm{C}$ NMR (of the mixture) $\delta 138.9,138.7$, $138.4,138.3,135.6,135.5,117.6,117.1,116.5,116.3,114.6$, $114.5,109.7$ (X2), 78.3, 78.2, 76.7, 76.1, 69.8, 69.7, 67.7 (X2), 45.7, 45.1, 36.7, 36.5, 36.1, 35.6, 35.3, 35.2, 33.6, 32.9, 29.7, 29.4, 25.3 (X2), 24.1 (X2), 24.0, 23.9; HRMS (ESI) $m / z$ calcd. for $\mathrm{C}_{20} \mathrm{H}_{32} \mathrm{O}_{3} \mathrm{Na}(\mathrm{M}+\mathrm{Na})^{+}, 343.2249$; Found, 343.2248 .

\section{7 (2S)-2-((3S)-5-((2-Methylallyl)oxy)nona-1,8- dien-3-yl)-1,4-dioxaspiro[4.5]decane $(9 \mathrm{c})$}

Following the same allylation procedure as described in the preparation of 9a, the allyl alcohol16 (137 $\mathrm{mg}, 0.49 \mathrm{mmol})$ was converted to the triene $9 f(103 \mathrm{mg}, 63 \%$, purified by column chromatography, $3 \% \mathrm{EA} / \mathrm{PE}$ as eluent) by using sodium hydride (47 mg, $0.98 \mathrm{mmol}, 50 \%$ suspension in oil) and 3-chloro-2-methylpropene (0.12 mL, $1.23 \mathrm{mmol}) ;{ }^{1} \mathrm{H} \mathrm{NMR}$ (of the mixture) $\delta 5.88-5.75(1 \mathrm{H}, \mathrm{m}), 5.61-5.46(1 \mathrm{H}, \mathrm{m})$, 5.11-4.75 (6H, m), 3.95-3.73 (4H, m), 3.68-3.62 (1H, m), 3.44-3.35 (1H, m), 2.49-2.32 (1H, m), 2.24-2.08 (3H, m), 1.98-1.81 (1H, m), 1.74 (3H, s), 1.60-1.57 (9H, m), 1.40$1.35(3 \mathrm{H}, \mathrm{m}) ;{ }^{13} \mathrm{C}$ NMR (of the mixture) $\delta 143.1,143.0,139.0$, 138.8, 138.5, 138.4, 117.6, 117.2, 114.6, 114.5, 111.9, 111.6, 109.8 (X 2), 78.4, 78.3, 76.7, 76.3, 72.8, 72.7, 67.8, 67.7, 45.7, 45.3, 36.7, 36.6, 36.2, 35.5, 35.4, 35.3, 33.5, 32.9, 29.7, 29.4, 25.4 (X 2), 24.2 (X 2), 24.0 (X 2), 20.0, 19.9.

\section{8 (3S,5S)-2-methyl-5-((S)-1,4-}

dioxaspiro[4.5]decan-2-yl)hepta-1,6-dien-3-ol (17a) and (3R,5S)-2-Methyl-5- $((S)-1,4-$ dioxaspiro[4.5]decan-2-yl)hepta-1,6-dien-3-ol (18)

Grignard reagent [prepared from the reaction of 2bromopropene $(0.5 \mathrm{~mL}, 5.58 \mathrm{mmol})$ with $\mathrm{Mg}$ (107 mg, $4.46 \mathrm{mmol}$ )] was added to a solution of the aldehyde $\mathbf{1 3}$ (500 mg, $2.23 \mathrm{mmol})$ in dry $\mathrm{THF}(20 \mathrm{~mL})$ at $0^{\circ} \mathrm{C}$. The reaction mixture was stirred for $12 \mathrm{~h}$ then quenched by saturated aqueous of $\mathrm{NH}_{4} \mathrm{Cl}$. The clear solution was decanted and concentrated. The residual mass was chromatographed by using PE-EA (15\%) as the eluent to afford the alcohol 17a (213 mg, 36\%, colourless oil) and 18 (237 mg, 40\%, colourless oil) as a light yellow oil. 17a: $[\alpha]_{\mathrm{D}}^{25}-4.8\left(c 0.75, \mathrm{CHCl}_{3}\right)$; ${ }^{1} \mathrm{H}$ NMR $\delta$ 5.64-5.51 (1H, m), 5.18-5.07 (2H, m), 4.98-4.97 (1H, m), 4.81-4.80 (1H, m), 4.12-4.09 (1H, m), 3.99-3.89 (2H, m), 3.68-3.61 (1H, m), 2.53-2.51 (1H, m), 2.46-2.36 $(1 \mathrm{H}, \mathrm{m}), 1.93-1.83(1 \mathrm{H}, \mathrm{m}), 1.74(3 \mathrm{H}, \mathrm{s}), 1.63-1.53(8 \mathrm{H}$, m), 1.39 (3H, brs); ${ }^{13} \mathrm{C} \mathrm{NMR} \delta 148.2,138.2,117.5,110.3$, 110.1, 78.3, 73.7, 68.3, 46.3, 38.6, 36.5, 35.4, 25.3, 24.1, 24.0, 18.4; HRMS (ESI) calcd for $\mathrm{C}_{16} \mathrm{H}_{26} \mathrm{O}_{3} \mathrm{Na}(\mathrm{M}+\mathrm{Na})^{+}$,
289.1780; found 289.1782. 18: $[\alpha]_{\mathrm{D}}^{25}+11.1\left(c 0.44, \mathrm{CHCl}_{3}\right)$; ${ }^{1} \mathrm{H}$ NMR $\delta$ 5.65-5.53 (1H, m), 5.12-5.05 (2H, m), 4.97-4.96 $(1 \mathrm{H}, \mathrm{m}), 4.87-4.86(1 \mathrm{H}, \mathrm{m}), 4.22(1 \mathrm{H}, \mathrm{t}, J=6.6 \mathrm{~Hz})$, 3.97-3.89 (2H, m), 3.65-3.58 (1H, m), 2.43 (1H, brs), 2.25$2.15(1 \mathrm{H}, \mathrm{m}), 2.02-1.93(1 \mathrm{H}, \mathrm{m}), 1.73(3 \mathrm{H}, \mathrm{s}), 1.70-1.67$ (1H, m), 1.61-1.58 (8H, m), 1.39-1.36 (2H, m); ${ }^{13} \mathrm{C}$ NMR $\delta$ $146.8,138.2,117.3,112.0,110.1,78.1,74.1,68.2,45.5$, 37.6, 36.6, 35.3, 25.3, 24.2, 24.0, 17.5; HRMS (ESI) $m / z$ calcd. for $\mathrm{C}_{16} \mathrm{H}_{26} \mathrm{O}_{3} \mathrm{Na}(\mathrm{M}+\mathrm{Na})^{+}$, 289.1780; Found 289 . 1782.

\section{9 (1S,4S)-2-Methyl-4-((S)-1,4- \\ dioxaspiro[4.5]decan-2-yl)cyclopent-2-enol (19)}

Following the RCM experimental procedure as described in the preparation of $\mathbf{1 0 a}$, the diene $\mathbf{1 7 a}(22 \mathrm{mg}, 0.09 \mathrm{mmol})$ was converted to the cyclopentenol 19 (colorless oil, $17 \mathrm{mg}$, $80 \%$, purified by column chromatography, $20 \% \mathrm{EA} / \mathrm{PE}$ as eluent) by using Grubbs 2nd generation catalyst G II ( $4.3 \mathrm{mg}$, $0.005 \mathrm{mmol}) ;[\alpha]_{\mathrm{D}}^{25}+36.0\left(c 1.4, \mathrm{CHCl}_{3}\right) ;{ }^{1} \mathrm{H} \mathrm{NMR} \delta 5.37$ $(1 \mathrm{H}, \mathrm{s}), 4.32(1 \mathrm{H}, \mathrm{t}, J=8.4 \mathrm{~Hz}), 4.15-4.09(1 \mathrm{H}, \mathrm{m}), 4.02$ $(1 \mathrm{H}, \mathrm{dd}, J=7.8,6.6 \mathrm{~Hz}), 3.56(1 \mathrm{H}, \mathrm{t}, J=7.8 \mathrm{~Hz}), 2.75-$ $2.69(1 \mathrm{H}, \mathrm{m}), 2.43(1 \mathrm{H}, \mathrm{d}, J=10.5 \mathrm{~Hz}), 2.32-2.22(1 \mathrm{H}$, $\mathrm{m}), 1.82-1.81(3 \mathrm{H}, \mathrm{m}), 1.70-1.53(8 \mathrm{H}, \mathrm{m}), 1.43-1.33(3 \mathrm{H}$, $\mathrm{m}) ;{ }^{13} \mathrm{C}$ NMR $\delta$ 145.1, 128.0, 109.9, 78.4, 76.2, 68.0, 45.7, 36.1, 35.0, 34.9, 25.3, 24.1, 23.9, 14.1; HRMS (ESI) $m / z$ calcd. for $\mathrm{C}_{14} \mathrm{H}_{22} \mathrm{O}_{3} \mathrm{Na}(\mathrm{M}+\mathrm{Na})^{+}, 261.1467$; Found 261 . 1466.

\subsection{0 (1R,4S)-2-Methyl-4-((S)-1,4- dioxaspiro[4.5]decan-2-yl)cyclopent-2-enol (20)}

Following the RCM experimental procedure as described in the preparation of 10a, the diene 18 (35 $\mathrm{mg}, 0.13 \mathrm{mmol})$ was converted to the cyclopentenol 20 (colourless oil, $23 \mathrm{mg}$, $75 \%$, purified by column chromatography, $20 \%$ EA/PE as eluent) by using Grubbs 2nd generation catalyst G II (5.6 mg, $0.007 \mathrm{mmol}) ;[\alpha]_{\mathrm{D}}^{25}+62.0\left(c \quad 0.89, \mathrm{CHCl}_{3}\right) ;{ }^{1} \mathrm{H} \mathrm{NMR} \delta$ $5.37(1 \mathrm{H}, \mathrm{s}), 4.63-4.61(1 \mathrm{H}, \mathrm{m}), 3.99-3.87(2 \mathrm{H}, \mathrm{m}), 3.55$ $(1 \mathrm{H}, \mathrm{t}, J=7.2 \mathrm{~Hz}), 3.00-2.96(1 \mathrm{H}, \mathrm{m}), 2.22-2.13(1 \mathrm{H}$, $\mathrm{m}), 1.92-1.84(1 \mathrm{H}, \mathrm{m}), 1.79(3 \mathrm{H}, \mathrm{s}), 1.61-1.35(11 \mathrm{H}, \mathrm{m})$; ${ }^{13} \mathrm{C}$ NMR $\delta 144.8,128.1,109.6,79.4,78.7,67.5,47.0$, 37.5, 36.5, 35.1, 25.4, 24.2, 24.0, 13.9; HRMS (ESI) $m / z$ calcd. for $\mathrm{C}_{14} \mathrm{H}_{22} \mathrm{O}_{3} \mathrm{Na}(\mathrm{M}+\mathrm{Na})^{+}, 261.1467$; Found 261 . 1469 .

\subsection{1 (S)-2-((3S,5S)-5-(allyloxy)-6-methylhepta-1,6- dien-3-yl)-1,4-dioxaspiro[4.5]decane (17b)}

Following the same synthetic procedure as described in the preparation of 9a, carbinol 17a $(60 \mathrm{mg}, 0.23 \mathrm{mmol})$ was converted to triene $\mathbf{1 7 b}$ ( $56 \mathrm{mg}, 80 \%$, purified by column chromatography, $5 \%$ diethyl ether/PE as eluent) by using sodium hydride (17 mg, $0.34 \mathrm{mmol}, 50 \%$ suspension in oil) and allyl bromide $(0.04 \mathrm{~mL}, 0.46 \mathrm{mmol}) ;[\alpha]_{\mathrm{D}}^{25}+3.4\left(c 0.89, \mathrm{CHCl}_{3}\right)$; ${ }^{1} \mathrm{H}$ NMR $\delta$ 5.94-5.81 (1H, m), 5.61-5.49 (1H, m), 5.26-5.18 
$(1 \mathrm{H}, \mathrm{m}), 5.12-5.08(3 \mathrm{H}, \mathrm{m}), 4.88(2 \mathrm{H}, \mathrm{s}), 3.96-3.88(3 \mathrm{H}$, $\mathrm{m}), 3.75-3.61(3 \mathrm{H}, \mathrm{m}), 2.55-2.45(1 \mathrm{H}, \mathrm{m}), 2.06-1.97(1 \mathrm{H}$, $\mathrm{m}), 1.66(3 \mathrm{H}, \mathrm{s}), 1.61-1.52(8 \mathrm{H}, \mathrm{m}), 1.38-1.32(3 \mathrm{H}, \mathrm{m})$; ${ }^{13} \mathrm{C}$ NMR $\delta 145.5,138.1,135.5,117.7,116.3,112.7,109.7$, 80.4, 78.2, 69.1, 67.6, 44.8, 36.5, 35.8, 35.3, 25.4, 24.1, 24.0, 17.2; HRMS (ESI) $m / z$ calcd. for $\mathrm{C}_{19} \mathrm{H}_{30} \mathrm{O}_{3} \mathrm{Na}(\mathrm{M}+\mathrm{Na})^{+}$, 329.2093; Found 329.2091.

\subsection{2 (S)-2-((3S,5R)-5-(Allyloxy)-6-methylhepta-1,6- dien-3-yl)-1,4-dioxaspiro[4.5]decane $(\mathbf{9 g})$}

Following the same synthetic procedure as described in the preparation of $\mathbf{9 a}$, the carbinol $18(72 \mathrm{mg}, 0.27 \mathrm{mmol})$ was converted to the triene $9 \mathrm{~g}(67 \mathrm{mg}, 81 \%$, purified by column chromatography, 5\% EA/PE as eluent) by using sodium hydride (20 mg, $0.41 \mathrm{mmol}, 50 \%$ suspension in oil) and allyl bromide $(0.05 \mathrm{~mL}, 0.54 \mathrm{mmol}) ;[\alpha]_{\mathrm{D}}^{25}+19.9\left(c 1.00, \mathrm{CHCl}_{3}\right)$; ${ }^{1} \mathrm{H}$ NMR $\delta$ 5.93-5.80 (1H, m), 5.58-5.46 (1H, m), 5.25-4.85 (6H, m), 3.94-3.83 (3H, m), 3.77-3.57 (3H, m), 2.11-1.96 (2H, m), 1.82-1.74 (1H, m), $1.64(3 \mathrm{H}, \mathrm{s}), 1.56-1.50(8 \mathrm{H}, \mathrm{m})$, 1.36-1.33 (2H, m); ${ }^{13} \mathrm{C}$ NMR $\delta 143.8,138.1,135.3,117.4$, $116.5,115.0,109.7,81.7,78.0,68.8,67.6,45.4,36.7,35.2$, 34.4, 25.3, 24.1, 24.0, 15.9; HRMS (ESI) $\mathrm{m} / \mathrm{z}$ calcd. for $\mathrm{C}_{19} \mathrm{H}_{30} \mathrm{O}_{3} \mathrm{Na}(\mathrm{M}+\mathrm{Na})^{+}, 329.2093$; Found: 329.2095.

\subsection{3 (6S)-2-Methyl-6-((S)-1,4-dioxaspiro[4.5]decan- 2-yl)octa-1,7-dien-4-ol (21)}

Following the same synthetic procedure as described for the synthesis of 15, the aldehyde 13 (150 $\mathrm{mg}, 0.67 \mathrm{mmol}$ ) was converted to the carbinol 21 (144 mg, 77\%, colourless oil purified by column chromatography, 20\% EA/PE as eluent) using 2-methylallylmagnesium chloride $(1.7 \mathrm{~mL}, 0.87 \mathrm{mmol}$, $0.5(\mathrm{M})$ in THF); ${ }^{1} \mathrm{H}$ NMR (of the mixture) $\delta 5.65-5.46(1 \mathrm{H}$, m), 5.14-5.02 (2H, m), 4.82-4.74 (2H, m), 3.96-3.74 (3H, m), 3.65-3.60 (1H, m), 2.49-2.30 (2H, m, ), 2.22-2.08 (2H, $\mathrm{m}), 1.82-1.75(1 \mathrm{H}, \mathrm{m}), 1.73-1.72(3 \mathrm{H}, \mathrm{m}), 1.60-1.55(9 \mathrm{H}$, $\mathrm{m}), 1.37-1.35(2 \mathrm{H}, \mathrm{m}) ;{ }^{13} \mathrm{C} \mathrm{NMR}$ (of the mixture) $\delta 143.0$, $142.9,138.3,138.1,117.3,117.0,113.2,113.1,110.0$ (X 2), 78.3, 78.1, 68.2, 68.1, 67.4, 67.0, 46.9, 46.2, 45.7, 45.6, 39.9, $39.8,36.6,36.4,35.3,35.2,25.2$ (X 2), 24.2, 24.1, 24.0 (X 2), 22.6, 22.5;

\subsection{4 (2S)-2-((3S)-5-(Allyloxy)-7-methylocta-1,7- dien-3-yl)-1,4-dioxaspiro[4.5]decane $(\mathbf{9 h})$}

Following the same synthetic procedure as described in the preparation of 9a, the carbinol $21(75 \mathrm{mg}, 0.27 \mathrm{mmol})$ was converted to the triene $\mathbf{9 h}(69 \mathrm{mg}, 80 \%$, purified by column chromatography, 5\% EA/PE as eluent) by using sodium hydride (20 mg, $0.4 \mathrm{mmol}, 50 \%$ suspension in oil) and allyl bromide $(0.05 \mathrm{~mL}, 0.53 \mathrm{mmol}) ;{ }^{1} \mathrm{H}$ NMR (of the mixture) $\delta$ 5.96-5.82 (1H, m), 5.64-5.44 (1H, m), 5.27-5.20 (1H, m), 5.12-5.02 (3H, m), 4.77-4.72 (2H, m), 4.10-3.85 (4H, m), 3.69-3.46 (2H, m), 2.52-2.34 (1H, m), 2.32-2.05 (2H, m), $1.89-1.84(1 \mathrm{H}, \mathrm{m}), 1.74(\mathrm{~s}) \& 1.71(\mathrm{~s})(3 \mathrm{H}), 1.59-1.55(9 \mathrm{H}$, $\mathrm{m}), 1.42-1.27(2 \mathrm{H}, \mathrm{m}) ;{ }^{13} \mathrm{C} \mathrm{NMR}$ (of the mixture) $\delta 143.1$, $142.8,138.5,138.4,135.6,135.5,117.7,116.8,116.4,116.3$, 112.9, 112.7, 109.8, 109.7, 78.5, 78.2, 76.1, 75.0, 69.9, 69.8, 67.8, 67.7, 45.6, 45.1, 43.1, 42.7, 36.7, 36.5, 36.4, 36.0, 35.3, 35.2, 25.3 (X 2), 24.1 (X 2), 24.0, 23.9, 23.1, 23.0; HRMS (ESI) $\mathrm{m} / z$ calcd. for $\mathrm{C}_{20} \mathrm{H}_{32} \mathrm{O}_{3} \mathrm{Na}(\mathrm{M}+\mathrm{Na})^{+}, 343.2249$; Found 343.2247.

\subsection{5 (2S)-2-((2S)-1-(3,6-Dihydro-2H-pyran-2-yl)but- 3-en-2-yl)-1,4-dioxaspiro[4.5]decane (11c)}

Following the RCM experimental procedure as described in the preparation of $\mathbf{1 0 a}$, the triene $\mathbf{9 c}(30 \mathrm{mg}, 0.10 \mathrm{mmol})$ was converted to the dihydropyran 11c (colourless oil, $18 \mathrm{mg}$, $65 \%$, purified by column chromatography, $6 \%$ EA/PE as eluent) by using Grubbs 1st generation catalyst G I (4.1 mg, $0.005 \mathrm{mmol}$ ); ${ }^{1} \mathrm{H}$ NMR (of the mixture) $\delta 5.81-5.75(1 \mathrm{H}, \mathrm{m})$, 5.72-5.67 (1H, m), 5.62-5.46 (1H, m), 5.14-5.03 (2H, m), 4.15-3.88 (4H, m), 3.68-3.45 (2H, m), 2.56-2.22 (2H, m), 2.04-1.93 (2H, m), 1.91-1.80 (1H, m), 1.59-1.56 (8H, m), $1.39-1.33(2 \mathrm{H}, \mathrm{m}) ;{ }^{13} \mathrm{C} \mathrm{NMR}$ (of the mixture) $\delta 138.2,138.1$, 126.6, 126.5, 124.6, 124.2, 117.5, 117.0, 109.8 (X 2), 78.3, $78.1,71.9,70.8,67.8,67.6,65.8$ (X 2), 45.2, 44.7, 37.9, 37.1, 36.7, 36.5, 35.3, 35.2, 31.7, 30.8, 25.3 (X 2), 24.2 (X 2), 24.1, 24.0; HRMS (ESI) $m / z$ calcd. for $\mathrm{C}_{17} \mathrm{H}_{26} \mathrm{O}_{3} \mathrm{Na}(\mathrm{M}+\mathrm{Na})^{+}$, 301.1780; Found, 301.1781.

\subsection{6 (2S)-2-((2S)-1-(2,3,4,7-Tetrahydrooxepin-2- yl)but-3-en-2-yl)-1,4-dioxaspiro[4.5]decane (11e)}

Following the RCM experimental procedure as described in the preparation of $\mathbf{1 0 a}$, the triene $9 \mathbf{e}(70 \mathrm{mg}, 0.22 \mathrm{mmol})$ was converted to the oxepene 11e (colourless oil, $42 \mathrm{mg}, 66 \%$, purified by column chromatography, $5 \% \mathrm{EA} / \mathrm{PE}$ as eluent) by using Grubbs 1st generation catalyst G I (10 mg, $0.011 \mathrm{mmol})$; ${ }^{1} \mathrm{H}$ NMR (of the mixture) $\delta 5.90-5.76(1 \mathrm{H}, \mathrm{m}), 5.68-5.48$ (2H, m), 5.15-5.03 (2H, m), 4.30-4.21 (1H, m), 4.05-3.88 (4H, m), 3.72-3.58 (1H, m), 2.47-2.25 (2H, m), 2.23-2.05 (2H, m), 2.02-1.77 (2H, m), 1.64-1.57 (9H, m), $1.39(2 \mathrm{H}$, brs); ${ }^{13} \mathrm{C}$ NMR (of the mixture) $\delta 138.4,138.3,131.7$ (X 2), 130.3, 130.2, 117.7, 116.8, 109.8 (X 2), 79.1, 78.3, 78.2 (X 2), 67.8, 67.7, 66.8, 66.6, 45.9, 45.1, 37.9, 37.3, 36.7, 36.6, 35.3 (X 2), 35.1, 33.6, 26.4, 25.8, 25.4 (X 2), 24.2 (X 2), 24.1 (X 2); HRMS (ESI) $m / z$ calcd. for $\mathrm{C}_{18} \mathrm{H}_{28} \mathrm{O}_{3} \mathrm{Na}(\mathrm{M}+\mathrm{Na})^{+}$, 315.1936; Found 315.1937.

\subsection{7 (2S)-2-((1S)-5-((2-Methylallyl)oxy)cyclohex-2- en-1-yl)-1,4-dioxaspiro[4.5]decane (10d)}

Following the RCM experimental procedure as described in the preparation of $\mathbf{1 0 a}$, the triene $9 \mathbf{d}(36 \mathrm{mg}, 0.11 \mathrm{mmol})$ was converted to the carbocycle 10d (colourless oil, $22 \mathrm{mg}, 69 \%$, purified by column chromatography, $5 \% \mathrm{EA} / \mathrm{PE}$ as eluent) by using Grubbs 1 st generation catalyst G I ( $5 \mathrm{mg}, 0.006 \mathrm{mmol})$; ${ }^{1} \mathrm{H}$ NMR (of the mixture) $\delta$ 5.74-5.67 (1H m,), 5.53-5.45 
(1H, m), 4.96 (1H, s), 4.85 (1H, s), 4.02-3.91 (4H, m), 3.743.53 (2H, m), 2.54-2.37 (2H, m), 2.32-2.11 (2H, m), 2.01$1.84(2 \mathrm{H}, \mathrm{m}), 1.73(3 \mathrm{H}, \mathrm{s}), 1.61-1.54(6 \mathrm{H}, \mathrm{m}), 1.40-1.16(3 \mathrm{H}$, $\mathrm{m}$ ); ${ }^{13} \mathrm{C}$ NMR (of the mixture) $\delta 142.9,142.8,127.1,126.8$, 126.6, 126.5, 111.9, 111.8, 109.4 (X 2), 78.5, 78.4, 74.0, 72.1, 72.0, 70.8, 67.3, 66.7, 39.8, 36.8, 36.4, 36.3, 35.2, 35.0, 32.4, 32.1, 31.4, 29.5, 25.3 (X 2), 24.1 (X 2), 24.0, 23.9, 19.7 (X 2); HRMS (ESI) $m / z$ calcd. for $\mathrm{C}_{18} \mathrm{H}_{28} \mathrm{O}_{3} \mathrm{Na}(\mathrm{M}+\mathrm{Na})^{+}$, 315.1936; Found, 315.1933.

\subsection{8 (2S)-2-((1S)-6-((2-Methylallyl)oxy)cyclohept-2-} en-1-yl)-1,4-dioxaspiro[4.5]decane (10f)

Following the RCM experimental procedure as described in the preparation of $\mathbf{1 0 a}$, the triene $\mathbf{9 f}(66 \mathrm{mg}, 0.20 \mathrm{mmol})$ was converted to the carbocycle $\mathbf{1 0 f}$ (colourless oil, $24 \mathrm{mg}, 40 \%$, purified by column chromatography, 4\% EA/PE as eluent) along with recovery of starting material $(13 \mathrm{mg}, 20 \%)$ by using Grubbs 1st generation catalyst G I ( $8.1 \mathrm{mg}, 0.01 \mathrm{mmol})$; ${ }^{1} \mathrm{H}$ NMR (of the mixture) $\delta: 5.88-5.68(1 \mathrm{H}, \mathrm{m}), 5.55-5.47$ (1H, m), 4.97 (1H, s), 4.87 (s, 1H), 4.09-3.97 (2H, m), 3.953.85 (2H, m), 3.75-3.63 (1H, m), 3.44-3.37 (1H, m), 2.54$2.17(3 \mathrm{H}, \mathrm{m}), 2.10-1.88(3 \mathrm{H}, \mathrm{m}), 1.75(3 \mathrm{H}, \mathrm{s}), 1.69-1.58(8 \mathrm{H}$, $\mathrm{m}), 1.49-1.33(3 \mathrm{H}, \mathrm{m}) ;{ }^{13} \mathrm{C} \mathrm{NMR}$ (of the mixture) $\delta 143.1$ (X2), 132.9, 132.7, 132.3, 131.7, 111.9, 111.5, 109.7, 109.6, 80.9, 78.6, 78.5, 75.9, 72.3, 71.8, 67.5, 67.1, 38.4, 36.6, 36.5, $36.5,35.6,35.2,35.1,33.5,32.3,31.7,25.4$ (X2), 24.2 (X2), 24.0, 23.9, 23.5, 22.9, 19.7 (X2); HRMS (ESI) $\mathrm{m} / \mathrm{z}$ calcd. for $\mathrm{C}_{19} \mathrm{H}_{30} \mathrm{O}_{3} \mathrm{Na}(\mathrm{M}+\mathrm{Na})^{+}, 329.2093$; Found, 329.2096.

\subsection{9 (S)-2-((2R,4S)-2-(Prop-1-en-2-yl)-2,3,4,7-}

tetrahydrooxepin-4-yl)-1,4-

dioxaspiro[4.5]decane (12g)

Following the RCM experimental procedure as described in the preparation of $\mathbf{1 0 a}$, the triene $9 \mathrm{~g}(27 \mathrm{mg}, 0.09 \mathrm{mmol})$ was converted to the oxepene $\mathbf{1 2 g}$ (colorless oil, $14 \mathrm{mg}, 56 \%$, purified by column chromatography, $5 \% \mathrm{EA} / \mathrm{PE}$ as eluent) by using Grubbs 1st generation catalyst G I (4 mg, $0.005 \mathrm{mmol}$ ); $[\alpha]_{\mathrm{D}}^{25}+13.1\left(c \quad 0.22, \mathrm{CHCl}_{3}\right) ;{ }^{1} \mathrm{H} \mathrm{NMR} \delta 5.88-5.80(1 \mathrm{H}$, $\mathrm{m},), 5.66(1 \mathrm{H}, \mathrm{d}, J=11.4 \mathrm{~Hz}), 4.95(1 \mathrm{H}, \mathrm{s}), 4.87(1 \mathrm{H}$, s), 4.30-4.22 (1H, m), 4.11-3.88 (4H, m), 3.70-3.68 (1H, m), 2.75-2.69 (1H, m), 2.04-1.99 (1H, m), $1.78(3 \mathrm{H}, \mathrm{s})$, 1.66-1.54 (9H, m), 1.40-1.39 (2H, m); ${ }^{13} \mathrm{C}$ NMR $\delta 146.3$, 132.1, 131.7, 110.9, 109.7, 84.2, 78.4, 67.2, 65.8, 42.0, 36.5, 35.4, 35.0, 25.4, 24.2, 24.0, 19.7; HRMS (ESI) $\mathrm{m} / \mathrm{z}$ calcd. for $\mathrm{C}_{17} \mathrm{H}_{26} \mathrm{O}_{3} \mathrm{Na}(\mathrm{M}+\mathrm{Na})^{+}, 301.1780$; Found 301.1783 .

\section{$2.20 \quad(S)-2-((2 R, 4 S)-2-(2-M e t h y l a l l y l)-2,3,4,7-$ tetrahydrooxepin-4-yl)-1,4- dioxaspiro[4.5]decane $(\mathbf{1 2 h})$}

Following the RCM experimental procedure as described in the preparation of $\mathbf{1 0 a}$, the triene $\mathbf{9 h}(60 \mathrm{mg}, 0.19 \mathrm{mmol})$ was converted to the oxepene $\mathbf{1 2 h}$ (colourless oil, $16 \mathrm{mg}, 29 \%$, purified by column chromatography, $6 \% \mathrm{EA} / \mathrm{PE}$ as eluent) by using Grubbs 1st generation catalyst G I (8 mg, $0.01 \mathrm{mmol})$; $[\alpha]_{\mathrm{D}}^{25}+14.1\left(c\right.$ 1.7, $\left.\mathrm{CHCl}_{3}\right) ;{ }^{1} \mathrm{H}$ NMR $\delta$ 5.87-5.79 $(1 \mathrm{H}, \mathrm{m})$, 5.68-5.64 (1H, m), $4.80(1 \mathrm{H}, \mathrm{s}), 4.74(1 \mathrm{H}, \mathrm{s}), 4.31-4.24(1 \mathrm{H}$, $\mathrm{m}), 4.10-3.94(3 \mathrm{H}, \mathrm{m}), 3.82-3.76(1 \mathrm{H}, \mathrm{m}), 3.67-3.62(1 \mathrm{H}$, $\mathrm{m}), 2.66-2.63(1 \mathrm{H}, \mathrm{m}), 2.38-2.31(1 \mathrm{H}, \mathrm{m}), 2.20-2.13(1 \mathrm{H}, \mathrm{m})$, 1.95-1.89(1H, m), 1.76 (3H, s), 1.64-1.59 (9H, m), 1.43-1.25 $(2 \mathrm{H}, \mathrm{m}) ;{ }^{13} \mathrm{C}$ NMR $\delta 143.0,132.2,131.6,112.5,109.7,80.0$, 78.4, 67.2, 66.1, 44.8, 42.1, 37.1, 36.5, 35.0, 25.4, 24.2, 24.0, 22.8; HRMS (ESI) $m / z$ calcd. for $\mathrm{C}_{18} \mathrm{H}_{28} \mathrm{O}_{3} \mathrm{Na}, 315.1936$; Found 315.1938.

\section{Results and Discussion}

We initially chose the triene 9a as a model for this investigation. The triene 9a was prepared from the known aldehyde $\mathbf{1 3}^{6}$ (Scheme 4). Addition of vinyl magnesium bromide to the aldehyde $\mathbf{1 3}$ gave the dienol 14 along with its C-3-epimer in 1:1 ratio. The pure dienol $\mathbf{1 4}$ was obtained in $35 \%$ yield through column chromatography. The dienol $\mathbf{1 4}$ was then converted to the triene 9a through etherification $(\mathrm{NaH}, 3$-chloro-2methyl propene). When the triene 9a in toluene was treated with G-I at rt, unexpectedly the carbocycle 10a (entry 1 in Table 1) was formed exclusively although formation of dihydrofuran from diene containing Me group on one of the alkene units is well precedented. ${ }^{7}$ This is in contrast to the RCM of the known triene $9 \mathrm{~b}$, prepared from 14, which gave the known oxacycle $\mathbf{1 1 b}^{8}$ exclusively (entry 2 in Table 1 ). This contrasting result led us to investigate RCM of the trienes $\mathbf{9 c}-\mathbf{h}$ to demonstrate the generality of this unusual reaction pattern.

Trienes 9c-h were prepared from the known aldehyde $\mathbf{1 3}$ as shown in Scheme 5. For synthesis of the trienes $9 \mathrm{c}$ and $9 \mathrm{~d}$, the aldehyde $\mathbf{1 3}$ was first reacted with allylmagnesium chloride to produce an inseparable mixture of the carbinols $\mathbf{1 5}$. The mixture of carbinols $\mathbf{1 5}$ on reaction of its sodium alkoxide with allyl bromide and 3-chloro-2-methylpropene provided the trienes 9c and 9d respectively in over all excellent yields. For synthesis of the trienes $9 \mathbf{e}$ and $9 \mathbf{f}$ the aldehyde $\mathbf{1 3}$ was allowed to react with 4-bromo butenyl magnesium bromide to produce an inseparable mixture of the known carbinols 16. $^{3 a}$ The latter on etherification with allyl bromide and 3-chloro-2-methylpropene gave $9 \mathbf{e}$ and $\mathbf{9 f}$ respectively. For synthesis of the triene $9 \mathrm{~g}$, the Grignard reagent generated from 2-bromopropene was added to the aldehyde 13 to produce a 1:1 mixture of the carbinols $17 \mathbf{a}$ and 18. Chromatographic purification led to isolation of the pure compounds $\mathbf{1 7 a}$ and $\mathbf{1 8}$ in $36 \%$ and $40 \%$ yields respectively. For stereochemical assignment both the dienols 17a and 18 was separately subjected to RCM with G-II as catalyst to produce the cyclopentenols 19 


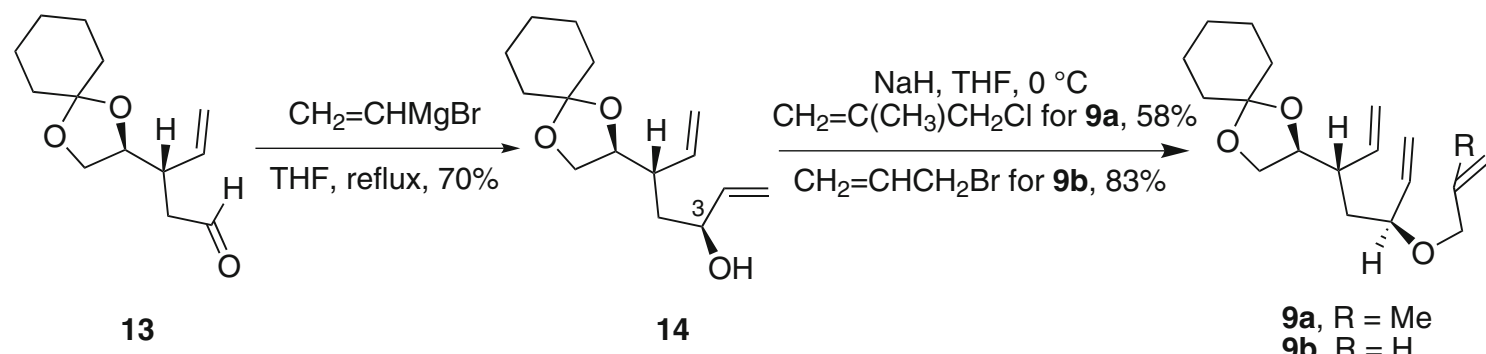

Scheme 4. Synthesis of the trienes $9 a$ and $9 b$.

and 20 in $80 \%$ and $75 \%$ yields respectively. Comparison of the 2D NMR spectra (HSQC, COSY and NOESY) of the cyclopentenols revealed that only the cyclopentenol obtained from the dienol $\mathbf{1 8}$ showed a cross peak between C-3 and C-5 H's enabling its stereochemical assignment as 20. Similar correlation was absent in the NOESY spectra of the cyclopentenol 19. This in turn dictated the stereochemical assignment of the dienols as 17a and 18. The carbinol 18 was then transformed to the triene $9 \mathrm{~g}$ through allylation of its alkoxide with allyl bromide. The triene $\mathbf{9 h}$ was prepared from the aldehyde $\mathbf{1 3}$ as follows. Reaction of the aldehyde $\mathbf{1 3}$ with methallyl magnesium chloride produced an inseparable mixture (1:1) of the carbinols 21 along with its C-4 epimer. This mixture was then transformed on reaction with allyl bromide to an inseparable mixture of the triene $\mathbf{9 h}$ and its C-4 epimer.

With the trienes $\mathbf{9 c}-\mathbf{h}$ ready in hand, RCM reaction was carried out with the catalyst G-I in toluene at rt. The trienes $9 \mathbf{c}$ and $9 \mathrm{e}$ without any Me substituent on any of the three alkene units afforded the dihydropyran $11 \mathrm{c}$ and the oxepene 11e in $65 \%$ and $66 \%$ yields respectively (entries 3 and 5 in Table 1). It may be noted that like the triene 9b, RCM of the trienes $9 \mathrm{c}$ and $9 \mathrm{e}$ produced oxacycles exclusively without any trace of the carbocycles 10c and 10e.

The scenario changed dramatically when a Me substituent was present on the alkene unit in the allyloxy group. The trienes 9d and 9f when subjected to RCM under the same reaction condition with G-I as catalyst, instead of the oxacycles 11d and 11f, the carbocycles 10d and 10 were obtained exclusively in $69 \%$ and $40 \%$ yield, respectively (entries 4 and 6 in Table 1). This indicates that the Me substituent on the alkene unit is playing a significant role in determining the reaction course. To get additional support about the influence of alkene substituent on the RCM reaction course, the trienes $9 \mathrm{~g}$ and $\mathbf{9 h}$ were subjected to RCM with G-I as catalyst. In both the case the oxepene derivative $\mathbf{1 2 g}$ and $\mathbf{1 2 h}$ was obtained respectively (entries 7 and 8 in Table 1). It is worthwhile to mention that unlike the
Table 1. RCM of acyclic trienes $\mathbf{9 a}-\mathbf{h}$

Entry Compound

smooth cyclisation of $\mathbf{9 g}$ to give the oxepene $\mathbf{1 2 g}$, the triene $\mathbf{1 7 b}$ obtained from the dienol 17a failed to cyclise and underwent decomposition under the reaction condition. The failure of $\mathbf{1 7 b}$ to undergo cyclisation is possibly due to the anti-disposition of the allyloxy group with the vinyl group. The stereochemical assignment to the oxepene 12h arising from RCM of the triene $9 \mathrm{~h}$ in the mixture is based on analogy to the formation of $\mathbf{1 2} \mathrm{g}$ from the triene $9 \mathrm{~g}$. The $\mathrm{C}-4$ epimer of the triene $9 \mathrm{~h}$ in the mixture failed to cyclise due to the anti-disposition of the allyloxy group with the vinyl group and decomposed. 

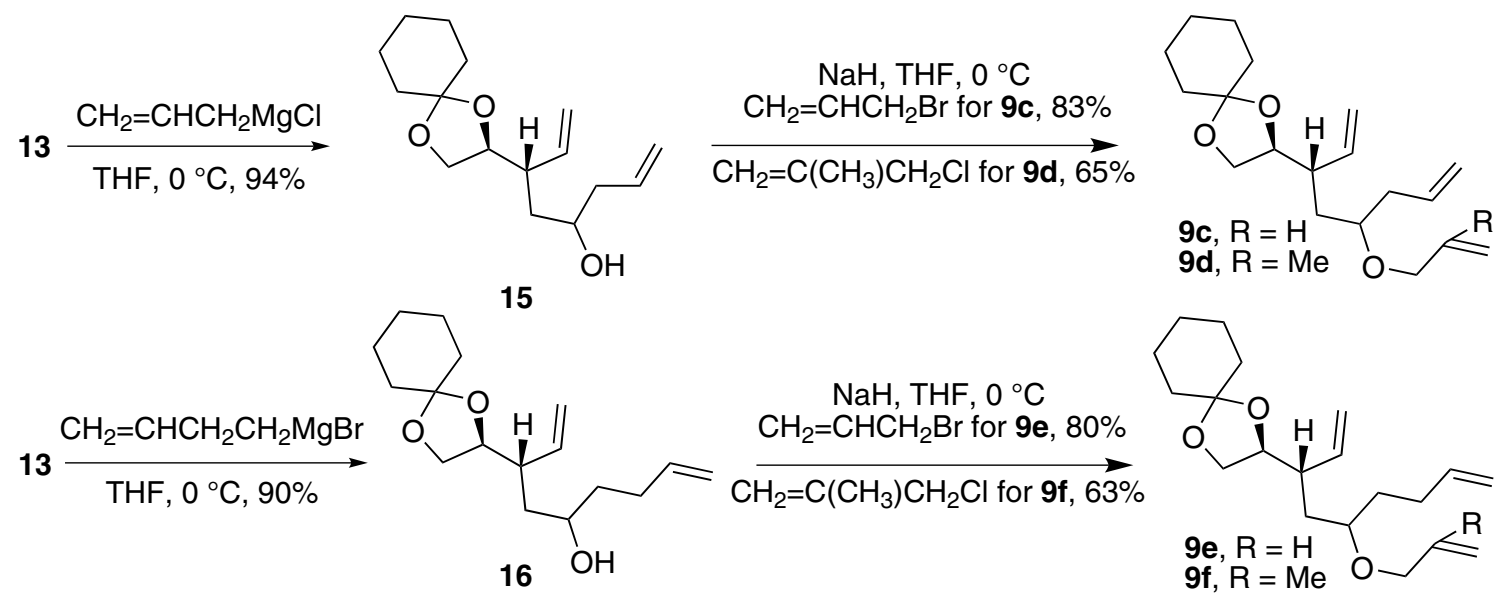

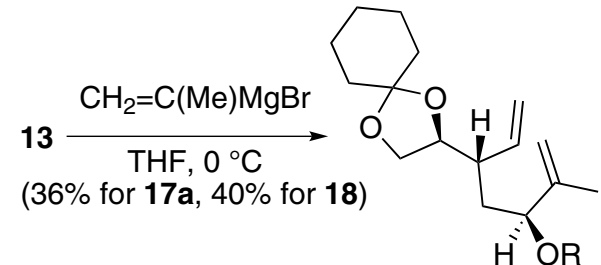

$17 a, \mathrm{R}=\mathrm{H}$

17b, $\mathrm{R}=\mathrm{CH}_{2} \mathrm{CH}=\mathrm{CH}_{2}$<smiles>CC1=C[C@H]([C@H]2COC3(CCCCC3)O2)C[C@@H]1O</smiles>

19

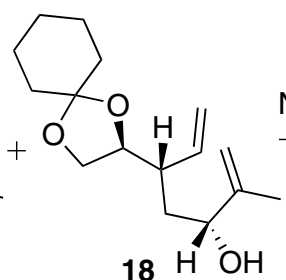

18
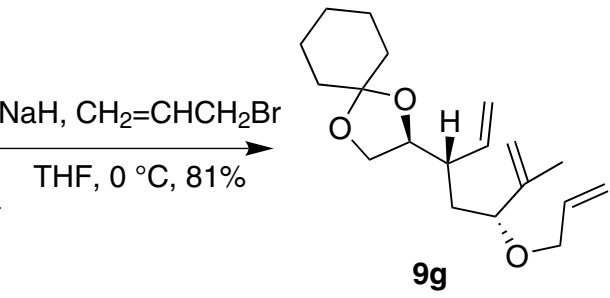

$9 g$

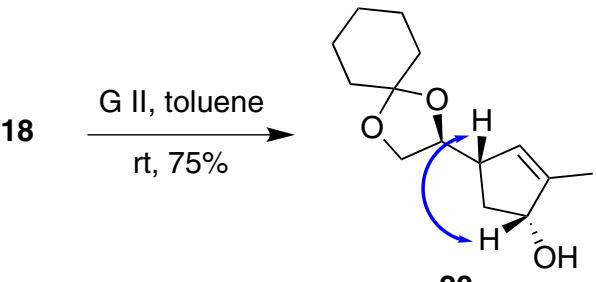

20

(ค) NOESY
$13 \underset{\mathrm{THF}, 0{ }^{\circ} \mathrm{C}, 77 \%}{\stackrel{\mathrm{CH}_{2}=\mathrm{C}(\mathrm{Me}) \mathrm{CH}_{2} \mathrm{MgCl}}{\longrightarrow}}$

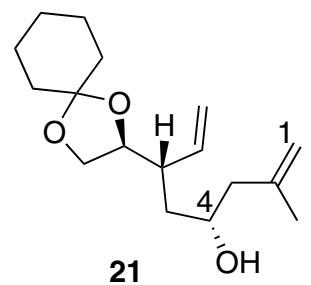

$\mathrm{NaH}, \mathrm{CH}_{2}=\mathrm{CHCH}_{2} \mathrm{Br}$

THF, $0{ }^{\circ} \mathrm{C}, 80 \%$

Scheme 5. Synthesis of the trienes $\mathbf{9 c - h}$.

\section{Conclusions}

This investigation demonstrates that factors such as nature and size of the rings to be formed during RCM with a given catalyst remaining the same, it is the substituent on the alkene that determines the reaction course to deliver either the carbocycle or the oxacycle. Further, strategically designed trienes with an alkyl group on the alkene unit can lead to the formation of either dihydrofuran or dihydropyran or oxepene, structural subunits present in a large number of natural products. ${ }^{9}$ Notably the oxacycles formed in this way contain a C-2 alkenyl chain which can further be employed for elaboration to natural products.

\section{Supplementary Information (SI)}

All additional information regarding characterisation of the new compounds using ${ }^{1} \mathrm{H}$ NMR, ${ }^{13} \mathrm{C}$ NMR and 2D NMR spectra are given in the supplementary information available at www.ias.ac.in/chemsci.

\section{Acknowledgements}

S.G. is grateful to Department of Science and Technology, Government of India and Indian National Science Academy for support of this work through J. C. Bose National Fellowship (Award No. SR/S2/JCB-83/2011) and INSA Senior Scientist program, respectively. R.D. thanks Council of Scientific and Industrial Research for a Senior Research Fellowship. 


\section{References}

1. For excellent accounts on RCM see: (a) Grubbs R H, Miller S J and Fu G C 1995 Ring-Closing Metathesis and Related Processes in Organic Synthesis Acc. Chem. Res. 28 446; (b) Fürstner A 1997 Recent advancements in Ring Closing Olefin Metathesis Top. Catal. 4 285; (c) Schuster M and Blechert S 1997 Olefin Metathesis in Organic Chemistry Angew. Chem. Int. Ed. 36 2036; (d) Grubbs R H and Chang S 1998 Recent Advances in Olefin Metathesis and Its Application in Organic Synthesis Tetrahedron 54 4413; (e) Armstrong S K 1998 Ring Closing Diene Metathesis in Organic Synthesis J. Chem. Soc. Perkin Trans. 1 371; (f) Fürstner A 2000 Olefin Metathesis and Beyond Angew. Chem. Int. Ed. 39 3012; (g) Kotha S and Sreenivasachary N 2001 Catalytic Metathesis Reaction in Organic Synthesis Indian J. Chem. B40 763; (h) Deiters A and Martin S F 2004 Synthesis of Oxygenand Nitrogen-Containing Heterocycles by Ring-Closing Metathesis Chem. Rev. 104 2199; (i) Nicolaou K C, Bulger P G and Sarlah D 2005 Metathesis Reactions in Total Synthesis Angew. Chem. Int. Ed. 44 4490; (j) Ghosh S, Ghosh S and Sarkar N 2006 Factors Influencing Ring Closure Through Olefin Metathesis-A Perspective J. Chem. Sci. 118 223; (k) Chattopadhyay S K, Karmakar S, Biswas T, Majumdar K C, Rahaman H and Roy B 2007 Formation of Medium-Ring Heterocycles by Diene and Enyne Metathesis Tetrahedron 633919

2. Ghosh S, Ghosh S and Sarkar N 2006 Factors Influencing Ring Closure Through Olefin Metathesis-A Perspective $J$. Chem. Sci. 118223

3. (a) Maity S, Matcha K and Ghosh S 2010 Synthetic Studies on Schisandra Nortriterpenoids. Stereocontrolled Synthesis of Enantiopure C-5-epi ABC Ring Systems of Micrandilactone A and Lancifodilactone G Using RCM J. Org. Chem. 75 4192; (b) Mondal S, Yadav R N and Ghosh S 2011 Influence of Diene Substituent Position on the Stereochemical Outcome in IMDA Reaction of Decatrienones. An Asymmetric Synthesis of $\mathrm{C}_{10}$-epiDihydro-epi-deoxy Arteannuin B Org. Lett. 13 6078; (c) Bose S, Ghosh M and Ghosh S 2012 Domino
Ring-Opening Metathesis-Ring-Closing Metathesis of Bicyclo[2.2.2] octene Derivatives: Scope and Limitations J. Org. Chem. 77 6345; (d) Datta R and Ghosh S 2017 Total Synthesis of the Marine Polyketide (-)-Gracilioether F J. Org. Chem. 827675

4. (a) Festa C, Lauro G, Marino S D, D'Auria M V, Monti M C, Casapullo A, D’Amore C, Renga, B, Mencarelli A, Petek S, Bifulco G, Fiorucci S and Zampella A 2012 Plakilactones from the Marine Sponge Plakinastrella mamillaris. Discovery of a New Class of Marine Ligands of Peroxisome Proliferator-Activated Receptor $\gamma$ J. Med. Chem. 55 8303; (b) Norris M D and Perkins M V 2016 Total Synthesis of Plakilactones C, B and des-Hydroxyplakilactone $\mathrm{B}$ by the Oxidative Cleavage of Gracilioether Furanylidenes J. Org. Chem. 816848

5. (a) Baylon C, Heck M-P and Mioskowski C 1999 Bis Ring Closing Olefin Metathesis for the Synthesis of Unsaturated Polycyclic Ethers. O-Membered Ring Cyclization in Favor of C-Membered Ring Cyclization J. Org. Chem. 64 3354; (b) Heck M-P, Baylon C, Nolan S P and Mioskowski C 2001 Triple Ring Closing Metathesis Reaction: synthesis of Adjacent Cyclic Ethers Org. Lett. 31989

6. Ghosh S, Bhaumik T, Sarkar N and Nayek A 2006 A Convenient Approach for Access to Both Carbapentofuranoses and Carbahexopyranoses. Stereocontrolled Synthesis of Enantiopure Carba-D-ribofuranoses, CarbaD-arabinofuranoses and Carba-L-gulopyranose J. Org. Chem. 719687

7. Kirkland T A and Grubbs R H 1997 Effects of Olefin Substitution on the Ring-Closing Metathesis of Dienes $J$. Org. Chem. 627310

8. Datta R, Sumalatha M and Ghosh S 2016 A Simple Approach to the Construction of the Core Structure Present in Bielschowskysin and Hippolachnin A J. Chem. Sci. 1281019

9. (a) Hoberg J O 1998 Synthesis of Seven-Membered Oxacycles Tetrahedron 54 12631; (b) Jacques R, Pal R, Parker N A, Sear C E, Smith P W, Ribaucourt A and Hodgson D M 2016 Recent Applications in Natural Product Synthesis of Dihydrofuran and -Pyran Formation by Ring-Closing Alkene Metathesis Org. Biomol. Chem. 145875 